\title{
SOHLH1 and SOHLH2 control Kit expression during postnatal male germ cell development
}

\author{
Florencia Barrios ${ }^{1, \star}$, Doria Filipponi ${ }^{2, \star}$, Federica Campolo ${ }^{1}$, Manuele Gori ${ }^{1}$, Federica Bramucci ${ }^{1}$, \\ Manuela Pellegrini ${ }^{1}$, Sergio Ottolenghi ${ }^{3}$, Pellegrino Rossi ${ }^{1}$, Emmanuele A. Jannini ${ }^{4}$ and Susanna Dolci ${ }^{1, \pm}$ \\ ${ }^{1}$ Dipartimento di Sanità Pubblica e Biologia Cellulare, Università di Roma 'Tor Vergata', Via Montpellier 1, Bldg. E Nord, Roma, 00133, Italy \\ ${ }^{2}$ Institute of Molecular and Cell Biology (IMCB), 61 Biopolis Drive, Proteos, 138673, Singapore \\ ${ }^{3}$ Dipartimento di Biotecnologie e Bioscienze, dell'Università degli Studi, Milano-Bicocca Piazza della Scienza 2, 20126, Milano, Italy \\ ${ }^{4}$ Dipartimento di Medicina Sperimentale, Università dell'Aquila, Via Giovanni Falcone 25, 67100 Coppito, L'Aquila, Italy \\ *These authors contributed equally to this work \\ ${ }^{\ddagger}$ Author for correspondence (dolci@uniroma2.it) \\ Accepted 8 November 2011 \\ Journal of Cell Science 125, 1455-1464 \\ (C) 2012. Published by The Company of Biologists Ltd \\ doi: 10.1242/jcs.092593
}

\section{Summary}

How Kit expression is regulated in the germline remains unknown. SOHLH1 and SOHLH2, two bHLH transcription factors specifically expressed in germ cells, are involved in spermatogonia and oocyte differentiation. In the male, deletion of each factor causes loss of Kitexpressing spermatogonia in the prepuberal testis. In the female, SOHLH1 and SOHLH2 ablations cause oocyte loss in the neonatal ovary. To investigate whether Kit expression is regulated by these two factors in male germ cells, we examined SOHLH1 and SOHLH2 expression during fetal and postnatal mouse development. We found a strong positive correlation between Kit and the two transcription factors only in postnatal spermatogonia. SOHLH2 was enriched in undifferentiated spermatogonia, whereas SOHLH1 expression was maximal at Kit-dependent stages. Expression of SOHLH1, but not SOHLH2, was increased in postnatal mitotic germ cells by treatment with all-trans retinoic acid. We found that E-box sequences within the Kit promoter and its first intron can be transactivated in transfection experiments overexpressing Sohlh1 or Sohlh2. Co-transfection of both factors showed a cooperative effect. EMSA experiments showed that SOHLH1 and SOHLH2 can independently and cooperatively bind an E-box-containing probe. In vivo coimmunoprecipitations indicated that the two proteins interact and overexpression of both factors increases endogenous Kit expression in embryonic stem cells. SOHLH1 was found by ChIP analysis to occupy an E-box-containing region within the Kit promoter in spermatogonia chromatin. Our results suggest that SOHLH1 and SOHLH2 directly stimulate Kit transcription in postnatal spermatogonia, thus activating the signaling involved in spermatogonia differentiation and spermatogenetic progression.

Key words: Kit, Sohlh1, Sohlh2, Germ cells

\section{Introduction}

The precursors of gametes, the primordial germ cells (PGCs), are first induced in the epiblast as a consequence of Bmp4 signaling from the extra-embryonic ectoderm (Ohinata et al., 2009). Following their specification, PGCs proliferate and migrate towards the sexually undifferentiated gonadal ridges. The proliferation and survival of PGCs in the embryonic gonad are dependent on the signaling pathway guided by kit ligand, which is produced by somatic cells, and its receptor, the tyrosine kinase Kit, in germ cells (Cairns et al., 2003; McLaren, 1998). From this early stage on, Kit signaling is essential for the survival and differentiation of male and female germ cells throughout their life span.

Kit is subject to tight transcriptional control because it is essential not only for germ cells but also for hematopoietic stem cell and melanocyte development. Indeed, a variety of transcription factors are known to regulate Kit expression in hematopoiesis and melanogenesis (reviewed by Sharma et al., 2006), but very little is known about the mechanisms that modulate Kit expression in the germline. In fetal oocytes, Kit is downregulated after meiotic entry, and is then upregulated perinatally to control oocyte growth. In fetal male germ cells, Kit is downregulated concomitantly with the start of the mitotic block that is maintained in gonocytes up to 2-3 days after birth. We have demonstrated that the repression of Kit expression is highly dependent on the action of PLZF (ZBTB16) (Filipponi et al., 2007) and that this prevents the depletion of the spermatogonia stem cell (SSC) pool (Buaas et al., 2004; Costoya et al., 2004).

To date, little is known about how Kit transcription is turned on during germ cell development in both sexes. What has been more extensively studied is the regulation of Kit expression in the hematopoietic and melanoblast lineages. TAL1 and MITF, two members of the Myc superfamily of bHLH transcription factors, have been shown to regulate Kit expression essential for hematopoietic stem cell and melanoblast proliferation, respectively (Krosl et al., 1998; Isozaki et al., 1994). bHLH transcriptional factors, especially those that are tissue specific, play important roles in cellular differentiation during various developmental stages of organogenesis (Massari and Murre, 2000). Sohlh1 and Sohlh2, two testis- and ovary-specific bHLH transcriptional factor genes, have recently been reported to be essential for both spermatogenesis (Ballow et al., 2006a) and oogenesis (Pangas et al., 2006). They share 50\% amino acid sequence identity, as well as a similar developmental expression pattern in both fetal and postnatal gonads (Ballow et al., 2006a; 
Ballow et al., 2006b; Pangas et al., 2006). Moreover, Sohlh1knockout and Sohlh2-knockout mice have common phenotypes, which in homozygosity lead to sterility in testis or ovaries (Ballow et al., 2006a; Ballow et al., 2006b; Hao et al., 2008; Pangas et al., 2006; Toyoda et al., 2009). In the female germline, through binding to canonical E-box sequences, SOHLH1 has been demonstrated to transcriptionally stimulate promoter activity of $L h x 8$ and $Z p l$, two genes crucial for the progression of primordial follicles into primary follicles (Pangas et al., 2006). Given that Sohlh2 is upregulated in Sohlh1-knockout animals (Ballow et al., 2006a), but the reciprocal upregulation does not occur in Sohlh2 knockouts (Hao et al., 2008; Toyoda et al., 2009), it has been hypothesized that SOHLH2 acts upstream of SOHLH1, potentially regulating its expression (Toyoda et al., 2009). Sohlh1-knockout (Pangas et al., 2006; Toyoda et al., 2009) and Sohlh2-knockout females show defects postnatally during early oogenesis, a process that strongly depends on Kit signaling (Toyoda et al., 2009). Interestingly, in postnatal male gonads, the differentiation of spermatogonia is also critically dependent on Kit signaling (Pellegrini et al., 2008) and is strongly disrupted in Sohlh1 (Ballow et al., 2006a) or Sohlh2 (Hao et al., 2008; Toyoda et al., 2009) knockout mice.

In this paper, we investigate whether a link between the two bHLH transcription factors and Kit exists in mouse spermatogonia. We found that SOHLH1 and SOHLH2 can regulate Kit promoter activity in transfection experiments. We further show that they can interact and bind E-box-containing sequences within the Kit promoter both in vitro and in vivo. Finally, we show that both SOHLH1 and SOHLH2 enhance endogenous Kit expression when transfected into embryonic stem (ES) cells. Since we found that SOHLH1 is upregulated by retinoic acid and is more abundant in Kit-positive spermatogonia, we speculate that this transcription factor initiates Kit expression together with SOHLH2 in undifferentiated spermatogonia and then maintains Kit expression in differentiating premeiotic germ cells.

\section{Results}

\section{Expression pattern of Sohlh1 and Sohlh2 in germ cells}

Ablation of either Sohlh1 or Sohlh2 affects the postnatal development of germ cells in a developmental window within which Kit expression is critical (Ballow et al., 2006a; Ballow et al., 2006b; Hao et al., 2008; Pangas et al., 2006; Toyoda et al., 2009). To verify the expression of SOHLH1 and SOHLH2 in spermatogonia we purified populations of Kit-negative (undifferentiated) and Kit-positive (differentiating) cells and found that SOHLH1 and SOHLH2 were both present in undifferentiated and differentiating spermatogonia (Fig. 1A). However, SOHLH1 was more abundant in Kit-positive cells, whereas SOHLH2 was enriched in Kit-negative spermatogonia. As a control for the purity of the spermatogonia population, cell extracts were probed for Kit and Stra8, which are known to be expressed in differentiating spermatogonia (Pellegrini et al., 2008).

In immunofluorescence experiments, SOHLH1 and SOHLH2 were localized on isolated spermatogonia or in whole seminiferous tubules obtained from p18 transgenic mice that express EGFP under the control of Kit regulatory regions (Cairns et al., 2003; Filipponi et al., 2007). Western blot analysis showed that SOHLH1 is expressed both in Kit-EGFP-negative and in some, but not all, Kit-EGFP-positive spermatogonia (Fig. 1B, top panels). Whole-mount preparations of seminiferous tubules confirmed that SOHLH1 was expressed in many Kit-EGFPpositive cells (Fig. 1B, left bottom panel). We estimated that $\sim 27 \%$ of total germ cells were EGFP negative and SOHLH1 positive, $55 \%$ were exclusively EGFP positive and $20 \%$ coexpressed both markers. In agreement with the western blot analysis, SOHLH2 was enriched among Kit-EGFP-negative spermatogonia (Fig. 1B, middle panels). Whole-mount preparations confirmed that SOHLH2 was mostly expressed in Kit-EGFP-negative cells (Fig. 1B, middle bottom panel), as was the case for the spermatogonial stem cell marker PLZF (Fig. 1B, right bottom panel). We estimated that $42 \%$ of total cells were EGFP negative and SOHLH2 positive, 52\% were exclusively EGFP positive and only $6 \%$ co-expressed both markers, suggesting that expression of SOHLH2 temporally precedes that of SOHLH1.

To examine whether Kit expression might be regulated by the two SOHLH factors during development, we studied the expression pattern of SOHLH1 and SOHLH2 in fetal gonads. SOHLH1 was not expressed early in female germ cell development, whereas in male germ cells it started to be expressed at $\sim 15.5$ days post-coitum (dpc), when gonocytes are found in mitotic block (Fig. 1B). SOHLH2 was expressed as early as $12.5 \mathrm{dpc}$ in female germ cells and 1 day later $(13.5 \mathrm{dpc})$ in male germ cells, and it was then continuously expressed, at least in the male, up to the perinatal period (Fig. 1C). This expression pattern did not overlap with that of Kit, which starts to be downregulated at $13.5 \mathrm{dpc}$ in both sexes (Fig. 1C), suggesting that Kit expression during fetal development is independent of SOHLH factor expression. By contrast, the expression of PLZF seemed to be in accordance with its postnatal role in spermatogonia. As expected for a transcriptional repressor (Filipponi et al., 2007), PLZF expression inversely correlated with Kit expression (Fig. 1C).

\section{AtRA upregulates SOHLH factors in postnatal spermatogonia}

We and others have demonstrated that all-trans retinoic acid (AtRA), an active analog of retinoic acid (RA), is able to strongly induce Kit expression in spermatogonia (Pellegrini et al., 2008; Zhou et al., 2008). To test whether AtRA is able to regulate SOHLH1 and SOHLH2 expression in spermatogonia cultures, we evaluated the levels of the two proteins after overnight stimulation with $0.3 \mu \mathrm{M}$ AtRA. SOHLH1 and Kit levels were strongly increased by the RA analog (Fig. 1D), whereas SOHLH2 levels were unaffected, further suggesting that SOHLH1 plays a role in differentiating spermatogonia.

\section{SOHLH1 and SOHLH2 activate transcription from $\mathrm{Kit}$ regulatory regions}

Our observation that expression of the SOHLH factors positively correlates with Kit expression postnatally, that SOHLH1 is upregulated by AtRA, and that Kit-positive spermatogonia are the only cell type affected in Sohlh1- or Sohlh2-knockout mice (Ballow et al., 2006a; Ballow et al., 2006b; Hao et al., 2008; Pangas et al., 2006; Toyoda et al., 2009), suggested that these two transcription factors could directly regulate Kit expression in spermatogonia. To test this hypothesis, we searched for canonical E-box sequences (CAGCTG and CACGTG) within the Kit promoter and within the first intron region that we have demonstrated is necessary to modulate Kit expression in the 
A

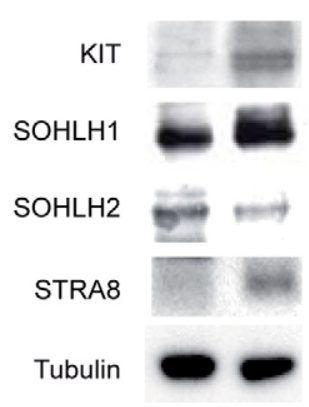

C female (dpc) $\begin{array}{lll}12.5 & 13.5 \quad 15.5\end{array}$ Kit

PLZF

\section{SOHLH1}

SOHLH2

Actin

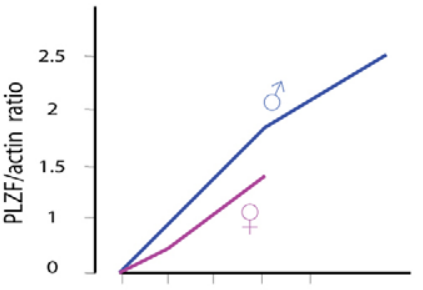

$\begin{array}{llllll}12.5 & 13.5 & 14.5 & 15.5 & 16.5 & 17.5\end{array}$

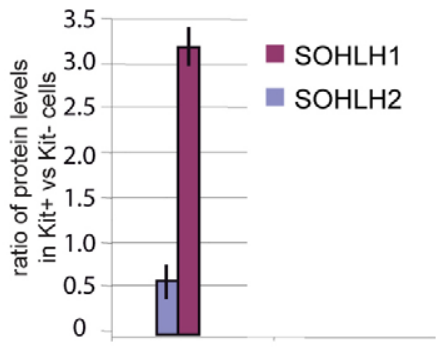

male (dpc)

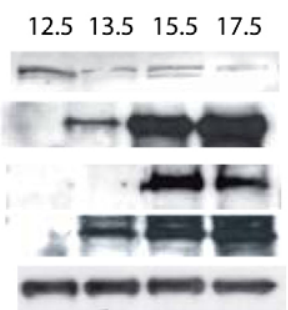

B
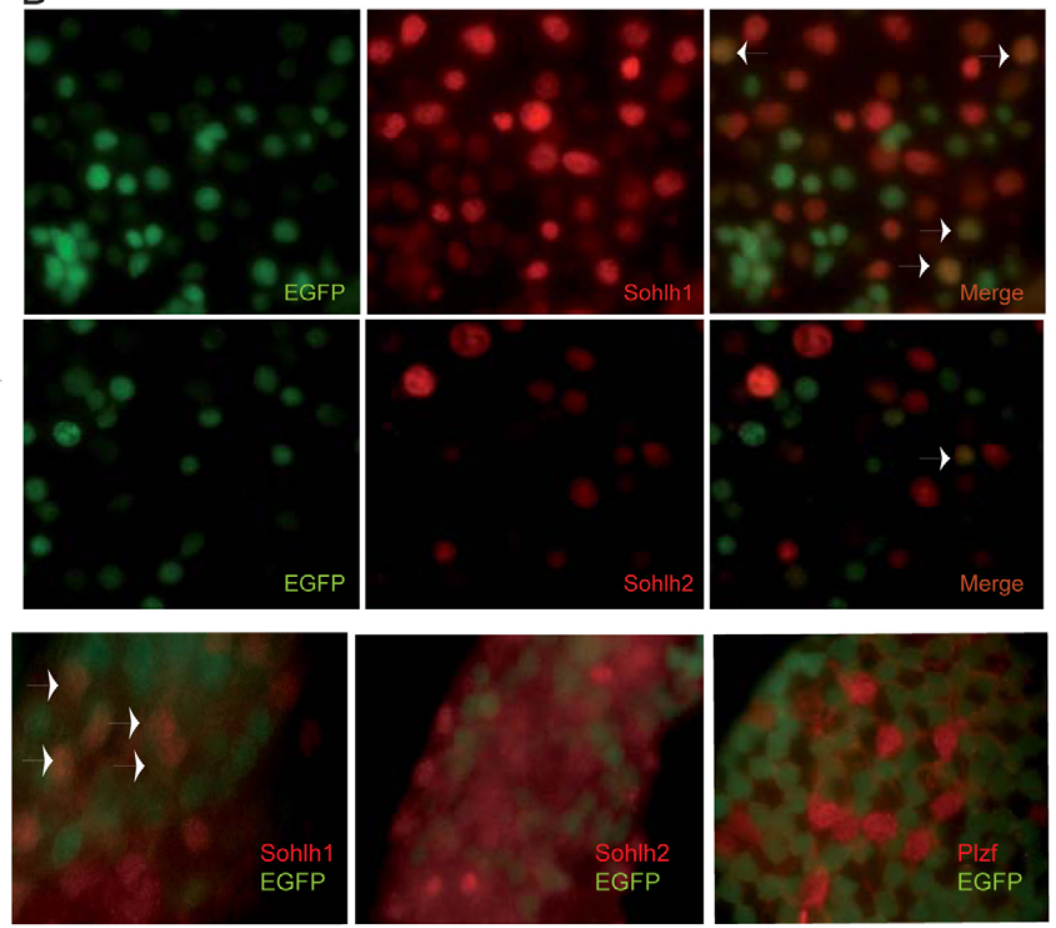

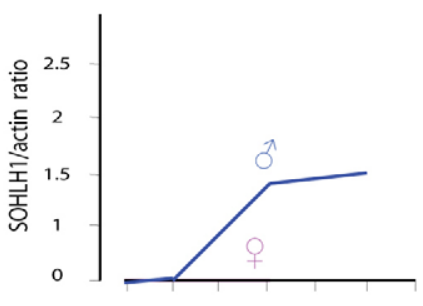

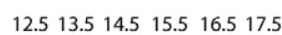

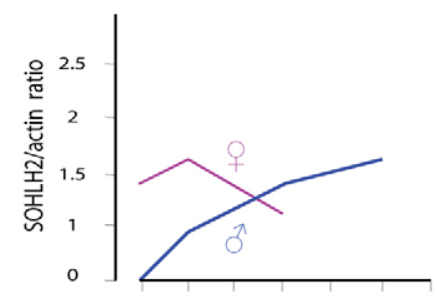

$\begin{array}{llllll}12.5 & 13.5 & 14.5 & 15.5 & 16.5 & 17.5\end{array}$

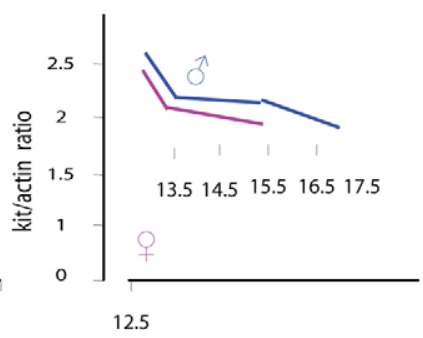

D

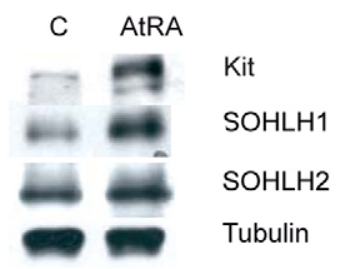

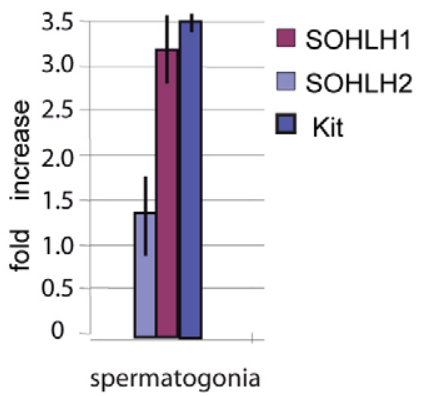

Fig. 1. Expression pattern of SOHLH1 and SOHLH2 in germ cells. (A) Western blot analysis (left) of Kit-positive and Kit-negative spermatogonia extracts from $7 \mathrm{dpp}$ mice probed for Kit, SOHLH1, SOHLH2 and Stra8. Tubulin was included as loading control. Bar graph (right) reports the ratio of the densitometric measurements of SOHLH1 and SOHLH2 levels (normalized for tubulin) in Kit-positive versus Kit-negative spermatogonia. The results represent the mean \pm s.d. of four independent experiments. (B) Top and middle rows show the immunolocalization of SOHLH1 and SOHLH2 (red), respectively, in isolated 7 dpp spermatogonia obtained from p18 transgenic mice that express EGFP (green) under the control of Kit regulatory regions. Left and middle panels in the bottom row show SOHLH1 and SOHLH2 immunolocalization (red), respectively, on whole-mount preparations of seminiferous tubules from 8 dpp p18 transgenic mice. Bottom right panel shows PLZF immunolocalization (red) on whole-mount preparations of seminiferous tubules from 8 dpp p18 transgenic mice. Arrowheads indicate EGFP-positive cells that co-express SOHLH1 or SOHLH2 (yellow-orange merge signal). (C) Western analysis for Kit, PLZF, SOHLH1, SOHLH2 and actin on female and male whole gonads isolated at the indicated fetal stages (dpc). Beneath are plotted the levels of PLZF, SOHLH1, SOHLH2 and Kit relative to actin in male and female gonads at the different developmental ages. Similar results were obtained in three independent experiments. (D). Western analysis (left) of Kit, SOHLH1, SOHLH2 and tubulin in $7 \mathrm{dpp}$ spermatogonia cultured overnight in the presence or absence of $0.3 \mu \mathrm{M}$ AtRA. To the right is shown the results of densitometric quantitation obtained from six independent experiments. Bars indicate relative fold increase \pm s.d. after AtRA stimulation. 
germline (Cairns et al., 2003; Filipponi et al., 2007). We took advantage of the previously characterized constructs used to generate the transgenic lines p18 and p13 (Fig. 2A) (Cairns et al., 2003; Filipponi et al., 2007). The p18 construct includes $\sim 7 \mathrm{~kb}$ of the Kit promoter, within which we found two CACGTG and four CAGCTG E-box sequences (Fig. 2A, yellow and red boxes, respectively), and $3.5 \mathrm{~kb}$ of the first Kit intron, which contains an E-box sequence of each type (Fig. 2A), driving the expression of an EGFP cassette (Cairns et al., 2003; Filipponi et al., 2007). We co-transfected p18 together with Sohh1-expressing or Sohlh2expressing plasmids, or both, into HEK293T cells, and evaluated the levels of EGFP expression by western blot analysis. To normalize for transfection efficiency, we introduced in all the samples a vector expressing a Myc-Tyr fragment, a construct that we previously validated to be constitutively expressed and inert (Filipponi et al., 2007). Interestingly, we observed that SOHLH1 or SOHLH2 was able to significantly enhance EGFP expression when compared with transfections that omitted the SOHLH expression plasmids (Fig. 2B). The upregulation of EGFP was further stimulated in a statistically significant fashion when both transcription factors were simultaneously introduced into the cells (Fig. 2B). These results suggested that SOHLH1 and SOHLH2 can independently stimulate Kit promoter activity, and also that their cooperation results in a stronger transactivation.

To investigate whether the E-boxes present in the Kit intron were essential for SOHLH responsiveness, we first co-transfected Sohlh1- or Sohlh2-expressing plasmids with the p13 construct, which contains only $6 \mathrm{~kb}$ of the Kit promoter (Fig. 2A) driving EGFP expression. As with the p18 construct, we found that SOHLH1 or SOHLH2, independently or together, can significantly upregulate EGFP expression (Fig. 2B). We then sequentially deleted sequences from the p13 construct to establish which of the six E-boxes in the promoter were crucial for SOHLH1 and/or SOHLH2 responsiveness. By digestion of p13 with appropriate restriction enzymes, the following constructs were generated: p316, which contains the two Eboxes of the Kit promoter proximal to the Egfp transcription start site; p178, which contains only the most proximal E-box; and p149, the basal promoter, which does not contain any E-boxes (Fig. 2A). When using p316 in co-transfection experiments, we found that both SOHLH1 and SOHLH2 were still able to
A

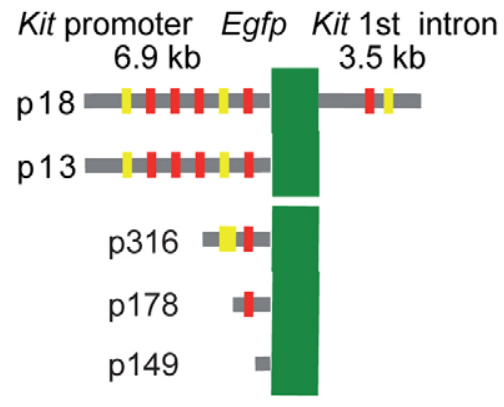

B

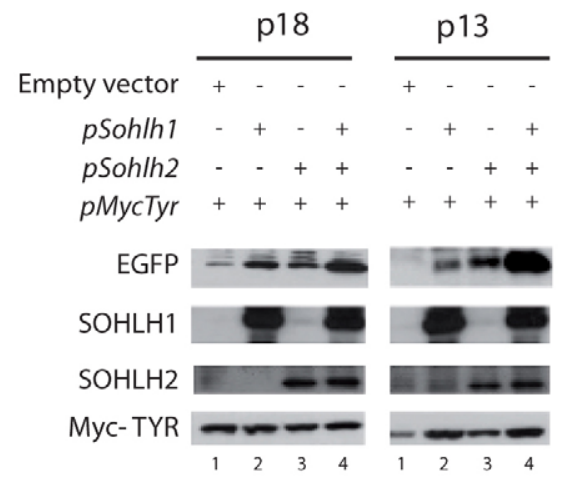

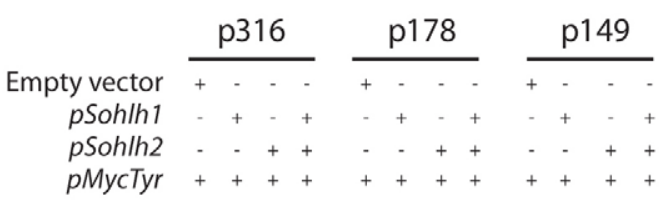

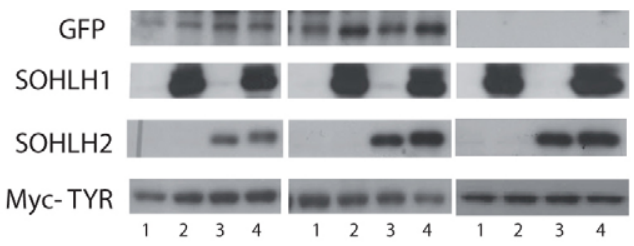

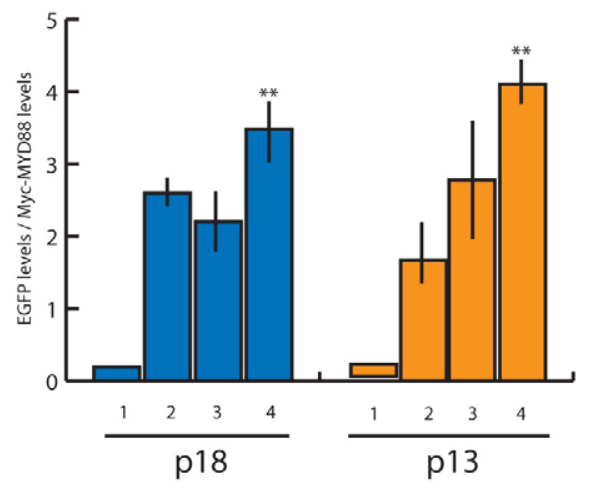

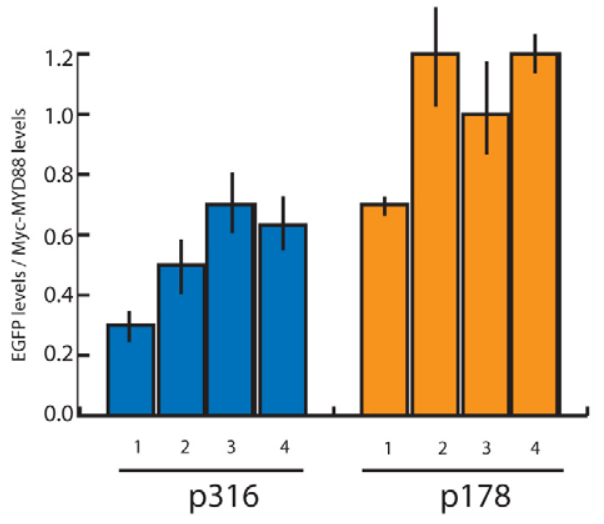

Fig. 2. SOHLH1 and/or SOHLH2 overexpression stimulates Kit promoter activity. (A) The constructs used in this study that contain Kit regulatory regions driving Egfp reporter gene expression (green box). The location of canonical Ebox consensus sequences is indicated: CAGCTG in red, CACGTG in yellow. (B) Co-transfection of the constructs shown in A with Sohlh1- and/or Sohlh2expressing vectors into HEK293T cells. Tir-Myc-expressing vector was cotransfected in all cases to normalize transfection efficiency. The bar charts show the relative levels of EGFP normalized for TIR-Myc. Results represent the mean \pm s.d. of at least three independent transfection experiments for each construct. $* * P<0.001$ for EGFP expression levels in Sohlh1 plus Sohlh2 co-transfected cell extracts versus extracts from single transfectants. 
upregulate EGFP expression; however, the cooperative effect between the two transcription factors was no longer observed. The same results were obtained with p178, which contains only one E-box. As expected, upon co-transfection with the basal promoter (p149) we did not detect EGFP expression in HEK293T cells, nor responsiveness to SOHLH1 or SOHLH2. Together, these results support the hypothesis that both SOHLH1 and SOHLH2 can independently and cooperatively transactivate Kit promoter in an heterologous system.

We then tested whether both proteins were able to enhance Kit expression in an in vivo model that is naturally prone to express Kit. We used the ES cell line R1, which exhibits basal Kit expression. The overexpression of either Sohlh1 or Sohlh2 strongly increased Kit levels. Interestingly, also in this in vivo model, co-transfection of both plasmids induced a consistent further increase in Kit expression (Fig. 3).

\section{SOHLH1 and SOHLH2 interact in vivo}

The cooperative stimulation of Kit promoter activity by both SOHLH1 and SOHLH2 suggested that these proteins might interact in vivo. We tested this hypothesis directly on postnatal spermatogonia. Cell extracts obtained from 7 days post-partum (dpp) male germ cells were immunoprecipitated with an antiSOHLH1 antibody and the immunocomplexes probed for SOHLH2. A fraction of the total amount of SOHLH2 was found in anti-SOHLH1 immunoprecipitates, but not in control IgG immunoprecipitates (Fig. 4), suggesting that the two proteins interact in vivo.

\section{Kit regulatory sequences are bound in vitro and in vivo by SOHLH factors}

Since we observed that E-box-containing regions were essential for SOHLH1 and SOHLH2 to drive EGFP expression, we evaluated whether these transcription factors can bind to Kit regulatory regions in vitro. We designed primers to amplify by PCR a 220 bp region that contains the two E-box sequences in the $3.5 \mathrm{~kb}$ of Kit intron 1 (Fig. 2A). The fragment was ${ }^{32} \mathrm{P}$-labeled in

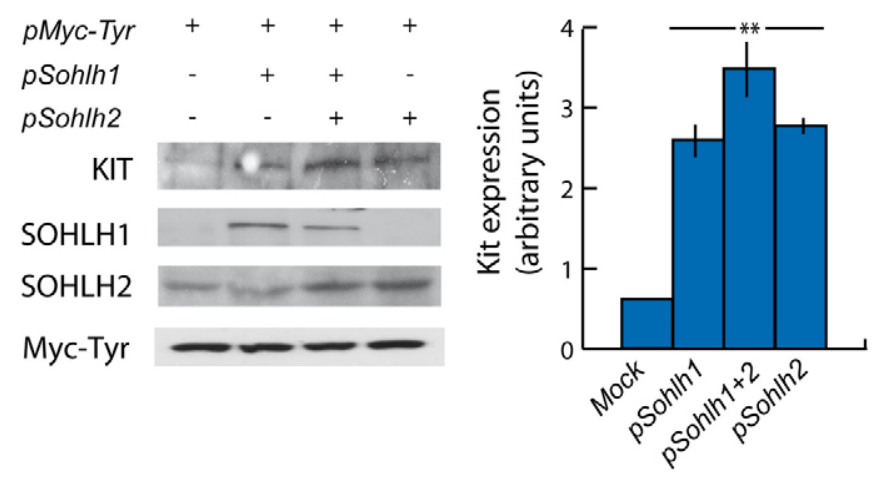

Fig. 3. Transfection of Sohlh1 and/or Sohlh2 stimulates endogenous Kit expression in ES cells. Western blot analysis of endogenous Kit expression in ES cells transfected with Sohlh1- and/or Sohlh2-expressing vector. Tir-Mycexpressing vector was co-transfected in all cases to normalize transfection efficiency. The bar chart shows the relative levels of endogenous Kit protein normalized for TIR-Myc. Results represent the mean \pm s.d. of three independent transfection experiments. ${ }^{* *} P<0.001$ for EGFP expression levels in Sohlh1 plus Sohlh2 co-transfected ES cell extracts versus extracts from single transfectants.

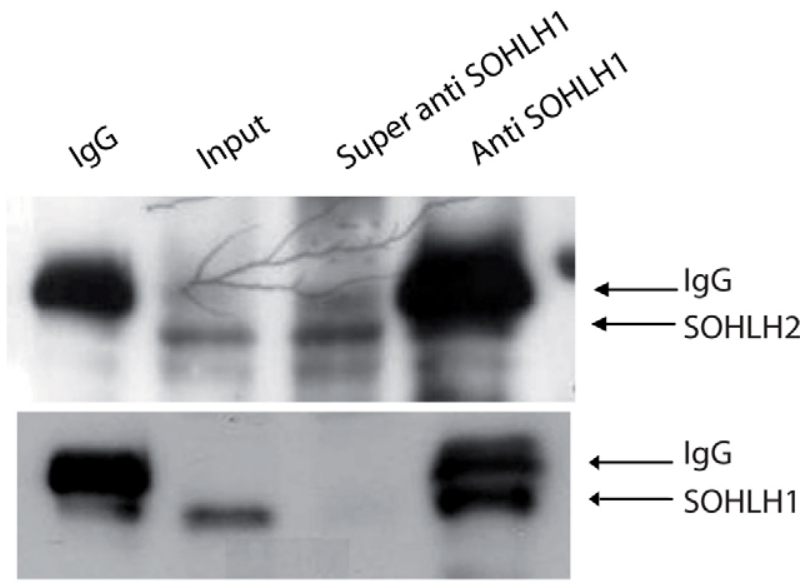

Fig. 4. SOHLH1 and SOHLH2 physically associate in postnatal spermatogonia. Protein extracts from 7 dpp spermatogonia were immunoprecipitated with anti-SOHLH1 antibodies. Subsequent western blot analyses of immunoprecipitates shows that a fraction of SOHLH2 is coimmunoprecipitated with SOHLH1.

order to perform an electromobility gel shift assay (EMSA) with nuclear extracts obtained from HEK293T cells transfected with vectors expressing Sohlh1, Sohlh2, or both (Fig. 5A). Nuclear extracts from Sohlhl-transfected cells showed three retarded bands, the slowest migrating of which was specific (lane 2), as shown by competition with an excess of cold probe (lane 3 ). Nuclear extracts from Sohlh2-transfected cells showed a prominent, specific, retarded band (lane 4), which was not observed in the presence of an excess of cold probe (lane 5). This retarded band showed a slower electrophoretic mobility than that obtained with SOHLH1-containing extracts. When both transcription factors were present, a retarded band was observed with an intermediate electrophoretic mobility (lane 6), which was faster than that generated by SOHLH2 and slower than that generated by SOHLH1. This band was not observed when the co-transfected extracts were primed in the presence of an excess of cold probe (lane 7), demonstrating the specificity of the protein-DNA interaction. When nuclear extracts of mocktransfected (pCMV-Egfp) HEK293T cells were primed with the labeled probe, two bands were observed (lane 8), which were competed by the cold probe (lane 9). The electrophoretic mobility of the two bands differed from those generated by the SOHLH1- and SOHLH2-transfected nuclear extracts, suggesting that other bHLH transcription factors are present in HEK293T cells. These results suggest that the two proteins can bind to the E-box sequences within Kit intron 1, possibly as homo- or heterodimers.

We also tested nuclear extract from $7 \mathrm{dpp}$ spermatogonia to assess the ability of endogenous SOHLH factors to bind to this Ebox sequence (Fig. 5B). The retarded bands (lane 2) were competed by preincubation with an excess of cold probe (lane 3 ), demonstrating the specificity of the DNA-protein complexes. The formation of this complex was prevented by preincubation of the extracts with an antibody against SOHLH1 (lane 4). The same antibody also prevented the formation of specific DNA-protein complexes in nuclear extracts from Sohlhl-transfected HEK293T cells (lane 6 versus lane 5). An antibody directed against an unrelated transcription factor $(\operatorname{Smad} 8)$ did not prevent the formation of the specific complexes (lane 7 versus lane 5). No 
A

$\begin{array}{rccccccccc}\text { Lane } & \mathbf{1} & \mathbf{2} & \mathbf{3} & \mathbf{4} & \mathbf{5} & \mathbf{6} & \mathbf{7} & \mathbf{8} & \mathbf{9} \\ \text { Probe } & + & + & + & + & + & + & + & + & + \\ \text { Cold probe } & - & - & + & - & + & - & + & - & + \\ \text { Hek-pSohlh1 } & - & + & + & - & - & + & + & - & - \\ \text { Hek-pSohlh2 } & - & - & - & + & + & + & + & - & - \\ \text { pEgfp } & - & - & - & - & - & - & - & + & +\end{array}$

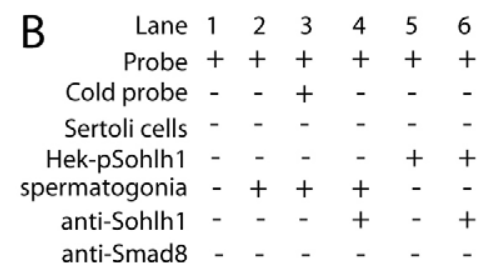

$\begin{array}{cccc}- & 8 & 9 & 10 \\ + & + & + \\ - & - & + \\ - & + & + \\ - & - & - \\ - & - & - \\ - & - & - \\ - & - & -\end{array}$
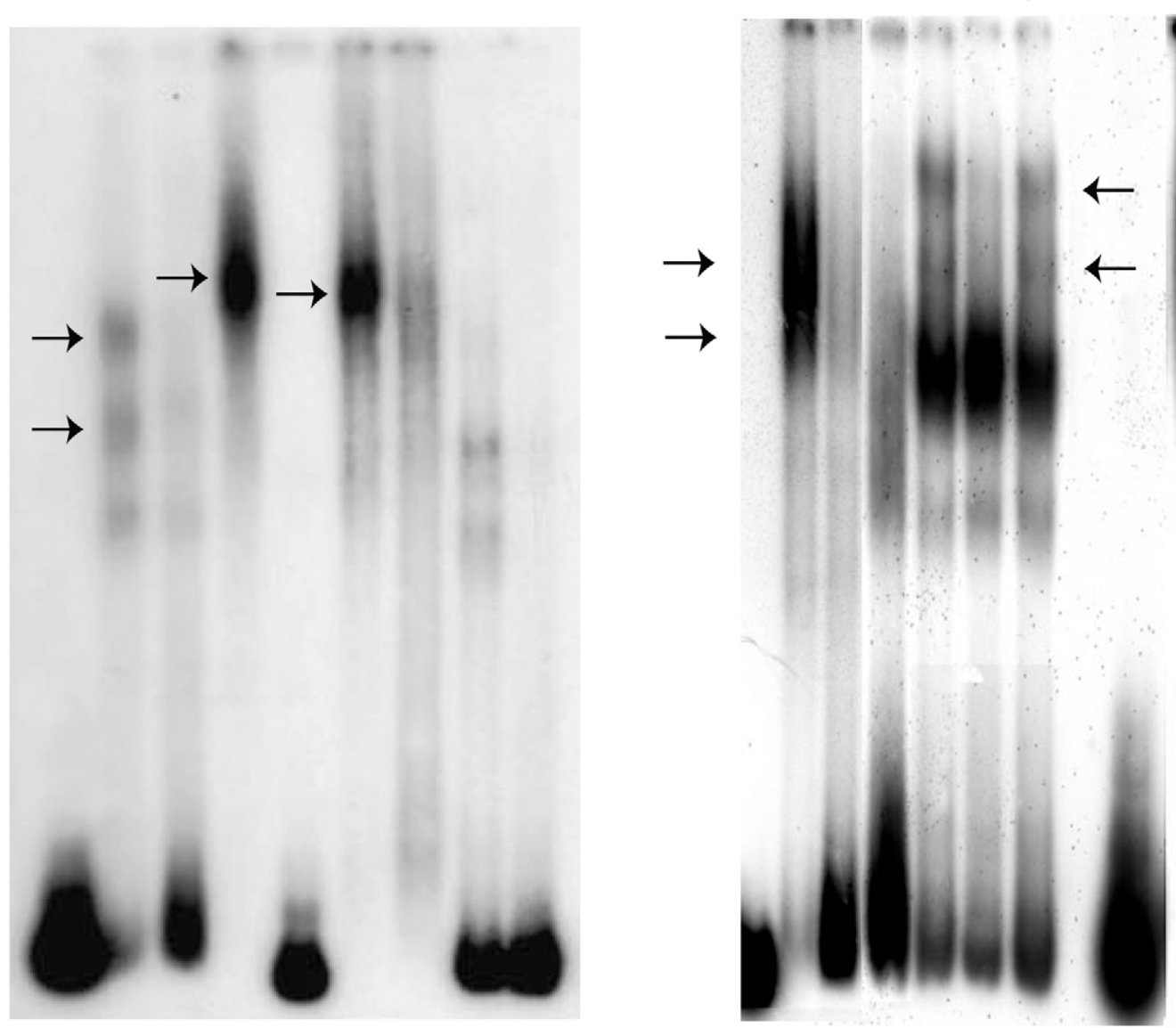

Fig. 5. SOHLH1 and SOHLH2 bind consensus E-box sequences within Kit regulatory regions. (A) EMSA of nuclear extracts from HEK293T cells transfected with Sohlh1-expressing (lanes 2, 3, 6,7) and/or Sohlh2-expressing (lanes 4-7) vectors, or with an empty vector (lanes 8, 9). The radiolabeled oligonucleotide used as a probe (lane 1) was obtained by PCR amplification of a sequence containing the two E-boxes within Kit intron 1 (region D as represented in Fig. 6A). A 100-fold molar excess of cold probe was included in lanes 3, 5, 7 and 9 for specific competition. (B) EMSA of nuclear extracts from 7 dpp spermatogonia (lanes 2-4), from HEK293T cells transfected with Sohlh1 expression vector (lanes 5-7) or from 7 dpp Sertoli cells (lanes 9, 10). The radiolabeled oligonucleotide (as in A) used as a probe was run in lanes 1 and 8. A 100-fold molar excess of cold probe was included in lanes 3 and 10 for specific competition. Anti-SOHLH1 (lanes 4, 6) or anti-Smad8 (lane 7) antibodies $(0.2 \mu \mathrm{g}$ ) were incubated at room temperature with nuclear extracts for 30 minutes before adding the radiolabeled probe. Arrows point to specific DNA-protein complexes.

specific bands were observed when the E-box-containing probe was primed with nuclear extracts obtained from 7 dpp Sertoli cell cultures (lanes 9 and 10).

To determine whether SOHLH factors were recruited to the endogenous Kit promoter in vivo, we performed ChIP experiments by immunoprecipitating SOHLH1 from formaldehyde cross-linked chromatin obtained from unselected $7 \mathrm{dpp}$ spermatogonia. We designed primer pairs that specifically amplify the single E-box sequences contained within the genomic region necessary for Kit expression in spermatogonia (Fig. 6A). By qPCR, only the CACGTG sequence immediately upstream of the Kit transcription start site (region B) was significantly enriched in SOHLH1-immunoprecipitated chromatin (Fig. 6B). Three other regions, containing no E-box sequences (region A), the most proximal E-box CAGCTG sequence (region C), or both E-boxes downstream from the Kit transcription start site (region D), were not amplified from SOHLH1-immunoprecipitated chromatin (Fig. 6B). To evaluate whether SOHLH1 binds in vivo to Kit promoter sequences in purified spermatogonia populations, we performed ChIP experiments on chromatin from both Kit-negative and Kit-positive spermatogonia. Region B was efficiently bound by SOHLH1 in chromatin from both Kitnegative (Fig. 6C) and Kit-positive (Fig. 6D) spermatogonia. Notably, SOHLH1 was also found to bind region C in chromatin from purified Kit-positive spermatogonia (Fig. 6D). These results demonstrate that both of the E-box sequences located just upstream of the Kit transcription start site are bound by SOHLH1 in vivo. 
A
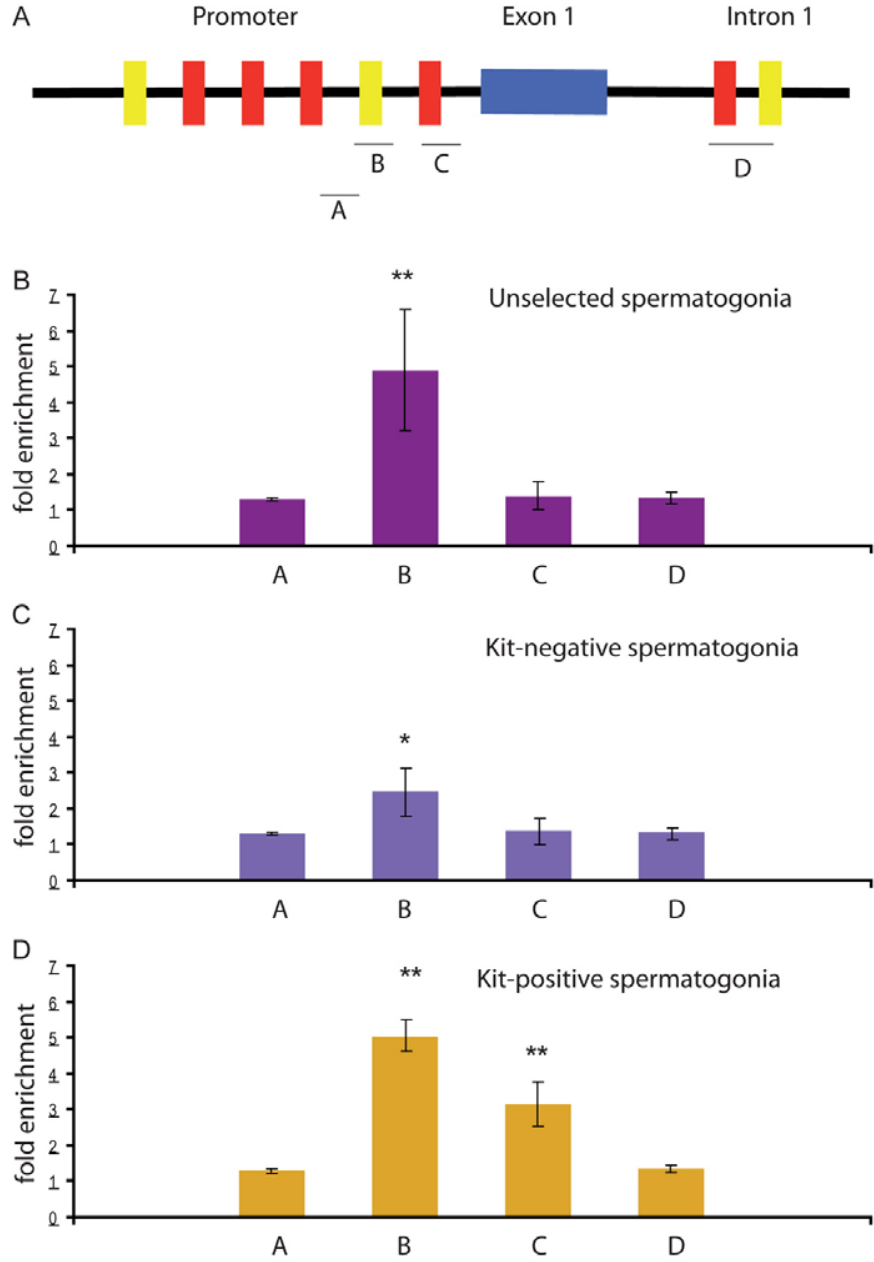

Fig. 6. SOHLH1 binds in vivo to a discrete E-box sequence within the Kit promoter. (A) Kit regulatory regions flanking exon 1. E-box sequences: CACGTG in yellow, CAGCTG in red. A, B, C and D represent the fragments amplified by qPCR after ChIP as shown in B-D. (B-D) ChIP experiments on unselected (B), Kit-negative (C) or Kit-positive (D) spermatogonia prepared from 7 dpp testes. Fold enrichment is shown after ChIP with anti-SOHLH1 antibodies of the indicated qPCR-amplified segments of the Kit promoter relative to ChIP with control IgGs. The data represent the mean \pm s.d. of three independent experiments. ${ }^{*} P<0.05, * * P<0.001$

\section{Discussion}

Unraveling the transcriptional network that controls the tissue and developmental expression pattern of Kit is of key importance, due to the fundamental role this gene has in many developmental processes. In the hematopoietic and melanoblast lineages, bHLH transcription factors such as TAL1 and MITF, respectively, have been shown to be essential for the control of Kit expression. Kit signaling is essential during fetal and postnatal development of the germline as well. Deletions or loss-of-function mutations in Kit lead to proliferative and survival defects of germ cells in both sexes. In contrast to the hematopoietic and melanoblast lineages, no bHLH factor had been demonstrated to be responsible for Kit expression in the germline. SOHLH1 and SOHLH2, two bHLH transcription factors that are specifically expressed in germ cells, had been hypothesized to be involved in the maintenance of Kitpositive postnatal germ cells of both sexes (Ballow et al., 2006a;
Toyoda et al., 2009). Indeed, Sohlh1 and Sohlh2 transcripts are present early during gametogenesis but targeted deletion of both genes, independently, leads to depletion of germ cells only postnatally.

To determine whether a regulatory correlation between Kit and SOHLH1 and/or SOHLH2 might exist, we studied the expression of these proteins in postnatal and fetal germ cells. In fetal germ cells, SOHLH1 was detectable from $13.5 \mathrm{dpc}$ in the male gonads, a developmental stage at which pro-spermatogonia enter mitotic block and Kit is downregulated. In the fetal ovaries, SOHLH1 was not detectable until $16.5 \mathrm{dpc}$. SOHLH2 expression was detectable before that of SOHLH1 in both female and male fetal germ cells (at 12.5 and $13.5 \mathrm{dpc}$, respectively). In males, however, Kit expression was downregulated at this stage and by $14.5 \mathrm{dpc}$ it was barely detectable. This evidence, and the observation that deletion of Sohlh1 or Sohlh2 does not impair the fetal development of germ cells, suggest that neither SOHLH factor is involved in regulating Kit expression in this developmental window. Alternatively, at least in the fetal testis, stimulation of Kit expression by SOHLH factors might be overcome by the parallel increase in the levels of PLZF, which we have shown to repress Kit transcription in undifferentiated spermatogonia (Filipponi et al., 2007). In the postnatal testis, we found expression of SOHLH1 and SOHLH2 in both Kit-negative and Kit-positive spermatogonia. SOHLH2 was preferentially expressed in undifferentiated spermatogonia, whereas SOHLH1 was increased in the Kit-positive cells. By immunofluorescence on whole-mount transgenic seminiferous tubules expressing EGFP under the control of Kit regulatory regions ( 18 construct), we found a consistent number of EGFPpositive cells that did not express SOHLH1. These cells are likely to represent early meiotic cells, such as preleptotene and leptotene spermatocytes, as judged by nuclear staining (data not shown). In these early meiotic cells, Kit expression is no longer present (Pellegrini et al., 2008) but the EGFP reporter is still present owing to the relatively high stability of this protein (our unpublished observations). Co-expression of the SOHLH factors in both undifferentiated and differentiating spermatogonia suggests that they might interact to upregulate Kit expression during the ongoing differentiation process. This hypothesis was strengthened by the finding that AtRA upregulates SOHLH1, as in the case for Kit, in cultured spermatogonia.

Either SOHLH1 or SOHLH2 is involved in the differentiation of Kit-positive spermatogonia and oocyte growth (Ballow et al., 2006a; Toyoda et al., 2009). It is well known that bHLHs function by forming homo- or heterodimers and binding to a consensus sequence termed the E-box (Massari and Murre, 2000). We speculated that the SOHLH factors might bind to the E-boxes contained within the regulatory regions of Kit and consequently transactivate the promoter. By computer-assisted analysis we found six canonical E-box sequences within the Kit promoter region (p13 plasmid) and two within a $3.5 \mathrm{~kb}$ region of Kit intron 1 (p18 plasmid). When we used these regions in transfection experiments, we found that both SOHLH1 and SOHLH2 independently increased the expression of the Egfp reporter gene in HEK293T cells. Furthermore, co-transfection of both transcription factors strongly increased EGFP expression. By sequentially deleting the E-box-containing regions within the intron and the promoter, responsiveness to SOHLH1 and SOHLH2 was completely abolished only when every E-boxcontaining region was deleted. The cooperative effect observed upon co-transfection of both SOHLH factors was abolished when 
only the two E-boxes within the Kit promoter that are most proximal to the transcription start site were left in the construct. These observations are in agreement with the recent finding that the human SOHLH1 gene, but not a deletion mutant associated to non-obstructive azoospermia, is able to transactivate the human KIT promoter in transfection experiments (Choi et al., 2010).

Moreover, our results suggest that multimeric SOHLH complexes are needed for optimal transcriptional activation of the Kit gene. By EMSA and co-immunoprecipitation, we tested whether the two proteins could interact. By gel shift analysis in a heterologous system, we found that both SOHLH1 and SOHLH2 produced two retarded bands when using a probe within Kit intron 1 containing two E-boxes. SOHLH1 was also able to bind to this Kit regulatory region in nuclear extracts obtained from $7 \mathrm{dpp}$ spermatogonia When co-expressed in HEK293T cells, SOHLH1 and SOHLH2 produced a third retarded band with an electrophoretic mobility consistent with heterocomplex formation. This possibility was also suggested by the observation that SOHLH2 associated with SOHLH1 in co-immunoprecipitations from spermatogonia extracts. The presence of residual SOHLH2, but not SOHLH1, in the supernatant of anti-SOHLH1 immunoprecipitates indicates that only a fraction of SOHLH2 protein is physically associated with SOHLH1. By ChIP analysis we found that the first of the two most proximal E-boxes present in the Kit 5'-flanking region was associated in vivo with SOHLH1 in chromatin from both Kit-negative and Kit-positive spermatogonia. Interestingly, SOHLH1 also bound the E-box-containing region

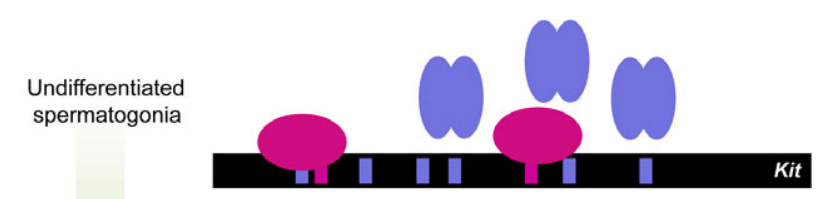

RA
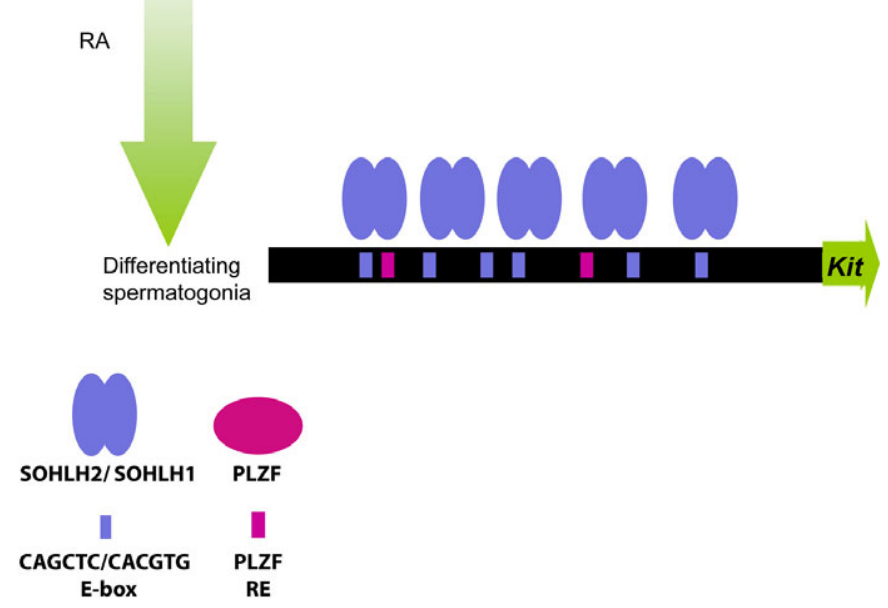

Fig. 7. The transcriptional network controlling Kit expression in postnatal spermatogonia. SOHLH1 and SOHLH2 form homo- and/or heterodimers that can bind to E-box elements within the Kit promoter region. In undifferentiated (Kit-negative) spermatogonia, PLZF binding to its cognate sites within the promoter prevents Kit transcription despite the presence of the SOHLH factors. Subsequent downregulation of PLZF allows progressive occupancy of the E-boxes by SOHLH2 and SOHLH1 complexes, initiating Kit transcription. In differentiating (Kit-positive) spermatogonia, SOHLH1, the expression of which is enhanced by retinoic acid (RA), becomes the main positive regulator of Kit transcription. RE, response element. immediately upstream of the Kit transcription start site in Kitpositive spermatogonia but not in Kit-negative cells. The ChIP data indicate that a progressive in vivo recruitment of SOHLH1 binding to the Kit promoter region occurs during spermatogonial differentiation, which parallels the increasing levels of Kit present in these cells. Although EMSA indicated that SOHLH1 and SOHLH2 can also bind to the intronic enhancer E-box sequences, SOHLH1 was not associated in vivo with this element, as assessed by ChIP experiments. However, as we did not obtain consistent results from ChIP analysis using SOHLH2 antibodies (data not shown), we cannot exclude the possibility that SOHLH2 associates in vivo with the E-boxes present within the first intron and/or with the other E-boxes present in the 5'-flanking region of the Kit gene.

Altogether, our results suggest that in postnatal spermatogonia SOHLH1 and SOHLH2 can form heterocomplexes on the Kit promoter in order to activate Kit transcription. We speculate that the presence of PLZF in undifferentiated spermatogonia (and possibly in fetal gonocytes) prevents Kit transcription in these cells, despite the fact that the SOHLH factors are already expressed. In line with this hypothesis is the observation that two PLZF-responsive elements are present within the Kit promoter closely flanking the first and the last E-boxes within the $6.9 \mathrm{~kb}$ fragment that we analyzed (Fig. 7). The downregulation of PLZF in early differentiating spermatogonia could then release cells from repression, allowing Kit expression to be primed by SOHLH2 and SOHLH1. Once Kit transcription is initiated by the cooperation of the two transcription factors, the presence of SOHLH1 and its upregulation by RA might then be sufficient to maintain elevated levels of Kit expression in differentiating spermatogonia.

\section{Materials and Methods}

\section{Transgenic mice}

We previously described the generation of transgenic mice carrying Enhanced green fluorescent protein (EGFP) as a reporter for the promoter and intronic activity of Kit (Cairns et al., 2003). In brief, a $\sim 7 \mathrm{~kb}$ fragment of the Kit promoter region was placed upstream of the Egfp reporter gene and used to generate the transgenic line p13 (Cairns et al., 2003). Another transgenic line, p18, was generated by adding $\sim 3.5 \mathrm{~kb}$ of Kit intron 1 to the 13 construct, downstream of the Egfp reporter (Cairns et al., 2003).

\section{Cell culture and transient transfection}

HEK293T cells were maintained in Dulbecco's Modified Eagle's Medium with $100 \mathrm{U} / \mathrm{ml}$ penicillin, $100 \mu \mathrm{g} / \mathrm{ml}$ streptomycin and $20 \mathrm{mM}$ glutamine. The D3 ES cell line was maintained in $\mathrm{KO}$ medium (Gibco, Milan, Italy) supplemented with $100 \mathrm{U} / \mathrm{ml}$ penicillin, $100 \mu \mathrm{g} / \mathrm{ml}$ streptomycin, $20 \mathrm{mM}$ glutamine, $1000 \mathrm{IU} / \mathrm{ml} \mathrm{LIF}$ (LIFES, Immunological Sciences, Rome, Italy). Transient transfections of HEK293T and D3 cell lines were performed using Lipofectamine according to the manufacturer's instructions (Invitrogen, Milan, Italy) with $1.5 \mu \mathrm{g}$ pcDNASohlh1 or pcDNA-Sohlh2 (kind gifts from Dr A. Rajkovic) or $0.75 \mu \mathrm{g}$ of each in the case of co-transfection, and $0.9 \mu \mathrm{g}$ for constructs containing the Kit regulatory regions (p13 or p18) (Filipponi et al., 2007), which were used only in HEK293T transfections. pCMV-TIR-myc plasmid (100 ng) (Loiarro et al., 2005) was included in the mixture as a control for transfection efficiency. pcDNA 3.1 (Invitrogen) was used as carrier to equalize the total amount of transfected DNA when pcDNA-Sohlh1 or pcDNA-Sohlh2 was omitted. In all co-transfection experiments, the total amount of transfected mammalian expression vectors was kept constant. All transfections were performed at least three times.

Postnatal male germ cells ( $7 \mathrm{dpp}$ ) were obtained as previously reported by sequential enzymatic digestion of testes from CD1 albino mice (Pellegrini et al., 2008). After preplating of cell suspensions, germ cells were cultured in Modified Earle's Medium (MEM, Gibco) with $20 \mathrm{mM}$ glutamine (Gibco), $2 \mathrm{mM}$ pyruvic acid (Sigma Aldrich, Milan, Italy), $1 \mathrm{mM}$ lactic acid (Sigma Aldrich), nonessential amino acids (Gibco), $100 \mathrm{U} / \mathrm{ml}$ penicillin and $100 \mu \mathrm{g} / \mathrm{ml}$ streptomycin (Gibco), without serum supplementation. Sertoli cell cultures were obtained as previously described (Pellegrini et al., 2008).

To obtain enriched fetal germ cell suspensions, fetal testes and ovaries were collected at different developmental stages and digested with trypsin (Gibco) and 
DNAse I (Sigma Aldrich). Cell suspensions were preplated in the same medium as postnatal male germ cells supplemented with $10 \%$ fetal bovine serum and recovered after 3 hours of culture to allow most of the somatic cells to adhere to the plastic dishes.

All-trans retinoic acid (Sigma) was dissolved in ethanol and used at a final concentration of $0.3 \mu \mathrm{M}$ (Pellegrini et al., 2008). Vehicle alone was added to the control cultures.

Separation of Kit-positive from Kit-negative spermatogonia was performed by magnetic-activated cell sorting (MACS) with CD117-conjugated microbead (Miltenyi Biotech, Germany) as previously described (Pellegrini et al., 2008).

\section{Immunofluorescence and whole-mounts}

For immunofluorescence, cells obtained from p18 transgenic mice were adhered onto poly-L-lysine-coated slides fixed in 4\% paraformaldehyde and permeabilized for 10 minutes in $0.1 \%$ Triton X-100 in phosphate-buffered saline (PBS). After a 1 -hour block in $5 \%$ bovine serum albumin (BSA) in PBS, anti-SOHLH1 (Ab 49272, Abcam, Cambridge, UK) or anti-SOHLH2 (a kind gift from Dr A Rajkovic) (Ballow et al., 2006b) antibodies were added at 1:100 dilution in 0.5\% BSA in PBS and incubated overnight at $4^{\circ} \mathrm{C}$. Cyanin-3-conjugated secondary antibodies were added to the cells for an additional hour. Nuclei were labeled by incubating the slides for 5 minutes in Hoechst $33349(1 \mu \mathrm{g} / \mathrm{ml})$. For wholemounts, seminiferous tubules from transgenic testes were fixed in $2 \%$ paraformaldehyde for 30 minutes and then permeabilized for 1 hour in $0.5 \%$ Triton X-100 in PBS. Tubules were then incubated with rabbit anti-SOHLH1 or rabbit anti SOHLH2 (a kind gift from Dr J. Miyazaki) (Toyoda et al., 2009) or antiPLZF (Chemicon, Milan, Italy) antibodies (1:100; or 1:200 in the case of rabbi anti-SOHLH2) overnight at $4{ }^{\circ} \mathrm{C}$ under constant rotation. After three washes in PBS containing $0.1 \%$ Triton $\mathrm{X}-100$, cyanin-3-conjugated secondary antibodies were added to the tubules for an additional hour. Nuclei were then counterstained in Hoechst 33349 .

\section{Electrophoretic mobility shift assay (EMSA)}

Nuclear extracts were obtained from $7 \mathrm{dpp}$ spermatogonia and from mockSohlh1- and/or Sohlh2-transfected HEK293T cells as previously reported (Filipponi et al., 2007). The probe containing one putative E-box (CACGTG) within Kit intron 1 was amplified from CD1 genomic DNA using the following primers: FW, TCTAGATTCTGGGAATCAGGGACT; RW, TCTAGACCTGAGTATGGCTATCA. The PCR product was purified using a kit from Roche digested with $X b a \mathrm{I}$ and cloned into pPCR-Script (Agilent Technologies). The fragment was subsequently excised and labeled with [ $\gamma$-32P]ATP (Perkin-Elmer). For binding reactions $(10 \mu \mathrm{l}), 10 \mu \mathrm{g}$ of nuclear extracts in binding buffer $(20 \mathrm{mM}$ HEPES pH 7.5, $1 \mathrm{mM} \mathrm{MgCl}, 10 \mu \mathrm{M} \mathrm{ZnCl}_{2}, 4 \%$ glycerol, $100 \mathrm{mg} / \mathrm{ml} \mathrm{BSA}$ ) were incubated on ice for 20 minutes in the presence or absence of a 100 -fold molar excess of unlabeled wild-type or mutated double-stranded oligonucleotides, or with anti-SOHLH1 or anti-Smad8 antibodies. The mixture was incubated for a further 20 minutes in the presence of $20,000 \mathrm{cpm}$ labeled probe at room temperature. DNA loading dye was added and samples run on a $0.5 \times$ Tris-borateEDTA nondenaturing polyacrylamide gel before autoradiography.

\section{Immunoprecipitation}

Spermatogonia were homogenized in lysis buffer $[100 \mathrm{mM} \mathrm{NaCl}, 10 \mathrm{mM} \mathrm{MgCl}$, $30 \mathrm{mM}$ Tris-HCl, $1 \mathrm{mM}$ DTT, protease inhibitor cocktail (Sigma-Aldrich)] supplemented with $0.5 \%$ Triton X-100. Soluble extracts were separated by centrifugation at $10,000 \mathrm{~g}$ for 10 minutes, and then were precleared for 1 hour on protein A-Sepharose beads (Sigma-Aldrich). After centrifugation for 1 minute at $1000 \mathrm{~g}$, supernatants were incubated with $2 \mu \mathrm{g}$ anti-SOHLH1 or rabbit IgGs (Sigma-Aldrich) overnight at $4{ }^{\circ} \mathrm{C}$ under constant rotation. Protein A-Sepharose beads were added to the immunocomplexes and incubated for an additional 2 hours at $4^{\circ} \mathrm{C}$. Beads were then washed three times with lysis buffer and eluted in SDS sample buffer for western blot analysis.

\section{Chromatin immunoprecipitation (ChIP)}

Proteins from at least $10^{7}$ isolated spermatogonia at $7 \mathrm{dpp}$ were cross-linked to DNA by direct addition to the culture medium of formaldehyde at $1 \%$ final concentration for 10 minutes at $37^{\circ} \mathrm{C}$. After sonication, a ChIP assay was performed according to the Abcam protocol. Protein-DNA complexes were immunoprecipitated overnight in the presence of the specific anti-SOHLH1 antibody or rabbit IgGs. DNA was purified using the QIAquick PCR purification kit (Qiagen, Milan, Italy) and resuspended in $1 \mathrm{ml} \mathrm{T}$ buffer $(10 \mathrm{mM}$ Tris-HC $\mathrm{pH} 8$ ) and used directly for qPCR. Real-time PCR was performed with $6 \mu$ DNA per reaction and $200 \mathrm{nM}$ primers, diluted to a final volume of $20 \mu \mathrm{l}$ in SYBR Green Reaction Mix (Applied Biosystems). Accumulation of fluorescent products was monitored using a 7300 Real-Time PCR System (Applied Biosystems) Each PCR reaction generated only the expected specific amplicon, as shown by the melting-temperature profiles of final products (dissociation curve, automatically measured). No PCR products were observed in the absence of template. Genomic regions of the Kit promoter and first intron indicated in Fig. 6A were amplified by
PCR using specific primers flanking the sites: A, fw 5'-TGAGTGCTATCACTTACGTC-3' and rv 5'-TGGCCCCGTGCTCACCATCC-3'; B, fw 5'-CACAAGATGATACACAAGCA- $3^{\prime}$ and rv 5'-ACTTACATGAATATGTGTGT-3'; C fw $5^{\prime}$-AGGTCCACGCTGGCCAATAG-3' and rv 5'-GGCACCCCTGACCTCAGTAA-3'; D, fw 5'-CAGCTGTTCTTGCCACCTCG-3' and rv 5'-GTATGGCTATCACGTGGGAG-3'

\section{Western blotting}

For western blot analysis cells were harvested and washed three times with icecold PBS. Cell lysis was performed with $10 \mathrm{mM}$ HEPES pH 7.9, 1\% Triton X-100, $10 \mathrm{mM} \mathrm{KCl}, 1.5 \mathrm{mM} \mathrm{MgCl}_{2}, 0.1 \mathrm{mM}$ EGTA, $0.5 \mathrm{mM}$ DTT, $10 \mathrm{mM} \beta-$ glycerophosphate, $0.1 \mathrm{mM}$ sodium vanadate and protease inhibitor cocktail (Sigma-Aldrich). Twenty to forty $\mu \mathrm{g}$ protein extract was separated by SDS PAGE in $10 \%$ polyacrylamide and transferred to PVDF membrane (Amersham, Piscataway, NJ, USA). The membrane was blocked in PBS with $0.1 \%$ Triton X100 (PBST) and 5\% fat-free milk powder for 1 hour at room temperature. Incubation with primary antibodies was carried out at $4{ }^{\circ} \mathrm{C}$ overnight in PBST with $5 \%$ BSA. Horseradish peroxidase-conjugated secondary antibody (Santa Cruz Biotechnology, Heidelberg, Germany) was used at 1:5000 dilution in PBST for 1 hour at room temperature. The horseradish peroxidase conjugate was detected by chemiluminescence with an ECL kit (Amersham, Milan, Italy) and autofluorography. Primary antibodies were: anti-SOHLH1 rabbit polyclonal (Abcam, 1:2000); anti-SOHLH2 (1:2000); anti-Kit rabbit polyclonal (Albanesi et al., 1996) (1:1000); anti-actin rabbit polyclonal (A2066, Sigma-Aldrich 1:1000); anti-EGFP rabbit polyclonal (Ab290, Abcam, 1:1000); and anti-myc epitope monoclonal (Santa Cruz, sc-2048, 1:1000). Images were quantitated by ImageQuant (GE Healthcare)

\section{Statistical analysis}

Student's $t$-test and analysis of variance (ANOVA) were used to assess significance. All experiments were performed at least three times or as stated in the figure legends. Continuous variables are summarized as mean and s.d.

\section{Acknowledgements}

We thank A. Rajkovic (Department of Obstetrics and Gynecology, Baylor College of Medicine, Houston, TX, USA) for plasmids and antibodies used throughout this study and J. Miyazaki (Osaka University Graduate School of Medicine, Osaka, Japan) for providing rabbit anti-SOHLH2 antibodies.

\section{Funding}

This work was supported by grants from the Ministero dell'università e della ricerca scientifica. [grant numbers Prin 2005059793_005, 200734H7WW_004, 200788TPYE_002, 20084XRSBS_004].

\section{References}

Albanesi, C., Geremia, R., Giorgio, M., Dolci, S., Sette, C. and Rossi, P. (1996). A cell- and developmental stage-specific promoter drives the expression of a truncated c-kit protein during mouse spermatid elongation. Development 122, 1291-1302.

Ballow, D., Meistrich, M. L., Matzuk, M. and Rajkovic, A. (2006a). Sohlh1 is essential for spermatogonial differentiation. Dev. Biol. 294, 161-167.

Ballow, D. J., Xin, Y., Choi, Y., Pangas, S. A. and Rajkovic, A. (2006b). Sohlh2 is a germ cell-specific bHLH transcription factor. Gene Expr. Patterns 6, 1014-1018.

Buaas, F. W., Kirsh, A. L., Sharma, M., McLean, D. J., Morris, J. L., Griswold, M. D., de Rooij, D. G. and Braun, R. E. (2004). Plzf is required in adult male germ cells for stem cell self-renewal. Nat. Genet. 36, 647-652.

Cairns, L. A., Moroni, E., Levantini, E., Giorgetti, A., Klinger, F. G., Ronzoni, S., Tatangelo, L., Tiveron, C., De Felici, M., Dolci, S. et al. (2003). Kit regulatory elements required for expression in developing hematopoietic and germ cell lineages. Blood 102, 3954-3962.

Choi, Y., Jeon, S., Choi, M., Lee, M. H., Park, M., Lee, D. R., Jun, K. Y., Kwon, Y., Lee, O. H., Song, S. H. et al. (2010). Mutations in SOHLH1 gene associate with nonobstructive azoospermia. Hum. Mutat. 31, 788-793.

Costoya, J. A., Hobbs, R. M., Barna, M., Cattoretti, G., Manova, K., Sukhwani, M., Orwig, K. E., Wolgemuth, D. J. and Pandolfi, P. P. (2004). Essential role of Plzf in maintenance of spermatogonial stem cells. Nat. Genet. 36, 653-659.

Filipponi, D., Hobbs, R. M., Ottolenghi, S., Rossi, P., Jannini, E. A., Pandolfi, P. P. and Dolci, S. (2007). Repression of kit expression by Plzf in germ cells. Mol. Cell. Biol. 27, 6770-6781

Hao, J., Yamamoto, M., Richardson, T. E., Chapman, K. M., Denard, B. S., Hammer, R. E., Zhao, G. Q, and Hamra, F. K. (2008). Sohlh2 knockout mice are male-sterile because of degeneration of differentiating type A spermatogonia. Stem Cells 26, 1587-1597.

Isozaki, K., Tsujimura, T., Nomura, S., Morii, E., Koshimizu, U., Nishimune, Y. and Kitamura, Y. (1994). Cell type-specific deficiency of c-kit gene expression in mutant mice of mi/mi genotype. Am. J. Pathol. 145, 827-836. 
Krosl, G., He, G., Lefrancois, M., Charron, F., Romeo, P. H., Jolicoeur, P., Kirsch, I. R., Nemer, M. and Hoang, T. (1998). Transcription factor SCL is required for ckit expression and c-Kit function in hemopoietic cells. J. Exp. Med. 188, 439-450.

Loiarro, M., Sette, C., Gallo, G., Ciacci, A., Fanto, N., Mastroianni, D., Carminati, P. and Ruggiero, V. (2005). Peptide-mediated interference of TIR domain dimerization in MyD88 inhibits interleukin-1-dependent activation of NF\{kappa\}B. J. Biol. Chem. 280, 15809-15814.

Massari, M. E. and Murre, C. (2000). Helix-loop-helix proteins: regulators of transcription in eucaryotic organisms. Mol. Cell. Biol. 20, 429-440.

McLaren, A. (1998). Germ cells and germ cell transplantation. Int. J. Dev. Biol. 42, 855-860.

Ohinata, Y., Ohta, H., Shigeta, M., Yamanaka, K., Wakayama, T. and Saitou, M. (2009). A signaling principle for the specification of the germ cell lineage in mice. Cell 137, 571-584.

Pangas, S. A., Choi, Y., Ballow, D. J., Zhao, Y., Westphal, H., Matzuk, M. M. and Rajkovic, A. (2006). Oogenesis requires germ cell-specific transcriptional regulators Sohlh1 and Lhx8. Proc. Natl. Acad. Sci. USA 103, 8090-8095.
Pellegrini, M., Filipponi, D., Gori, M., Barrios, F., Lolicato, F., Grimaldi, P., Rossi, P., Jannini, E. A., Geremia, R. and Dolci, S. (2008). ATRA and KL promote differentiation toward the meiotic program of male germ cells. Cell Cycle 7, 38783888 .

Sharma, S., Gurudutta, G. U., Satija, N. K., Pati, S., Afrin, F., Gupta, P., Verma, Y. K., Singh, V. K. and Tripathi, R. P. (2006). Stem cell c-KIT and HOXB4 genes: critical roles and mechanisms in self-renewal, proliferation, and differentiation. Stem Cells Dev. 15, 755-778.

Toyoda, S., Miyazaki, T., Miyazaki, S., Yoshimura, T., Yamamoto, M., Tashiro, F., Yamato, E. and Miyazaki, J. (2009). Sohlh2 affects differentiation of KIT positive oocytes and spermatogonia. Dev. Biol. 325, 238-248.

Zhou, Q., Li, Y., Nie, R., Friel, P., Mitchell, D., Evanoff, R. M., Pouchnik, D., Banasik, B., McCarrey, J. R., Small, C. and Griswold, M. D. (2008). Expression of stimulated by retinoic acid gene 8 (Stra8) and maturation of murine gonocytes and spermatogonia induced by retinoic acid in vitro. Biol. Reprod. 78, 537-545. 